\title{
Dihedral Angles of Convex Polyhedra
}

\author{
J.-M. Schlenker \\ Topologie et Dynamique, Départment de Mathématiques, \\ UMR 8628, Bât. 425, Université de Paris-Sud, \\ 91405 Orsay Cedex, France \\ jean-marc.schlenker@math.u-psud.fr \\ http://www.math.u-psud.fr/ schlenker
}

\begin{abstract}
Convex polyhedra in $H^{3}$ are not determined by (their combinatorics and) their edge lengths. Convex space-like polyhedra in the de Sitter space $S_{1}^{3}$ are determined neither by their dihedral angles nor by their edge lengths. The same holds of convex polyhedra in $S^{3}$.
\end{abstract}

\section{Introduction}

Let $P$ be a compact convex polyhedron in hyperbolic 3 -space $H^{3}$. It is classical to ask:

Question 1. Is $P$ determined, among convex polyhedra, by its combinatorics and its dihedral angles?

In other terms, if another convex polyhedron $Q$ has the same dihedral angles as $P$, is it congruent to $P$ ? This question also has an infinitesimal counterpart:

Question 1'. Does $P$ have a non-trivial infinitesimal deformation which does not change its dihedral angles?

Questions 1 and 1' can be related to interesting problems in hyperbolic geometry. Let $M$ be a hyperbolic 3-manifold, with cone singularities along geodesic segments (which might meet at "vertices") and with angle below $2 \pi$ around each singular segment. It would be interesting to know whether $M$ can be deformed—among hyperbolic manifolds with the same kind of singularities - without changing the angles around the singular loci. The answer is negative when the singularities are along closed geodesics which do not 
intersect (see $[\mathrm{HK}]$ ) but the general case is open. This question arises when one tries to put a hyperbolic metric on a manifold by deforming a hyperbolic cone metric, following the ideas of Thurston.

If $P$ is a convex hyperbolic polyhedron, one can take two copies of its inside and glue them along their corresponding faces; this produces a hyperbolic cone-manifold with vertices, and the convexity of $P$ means that the angle along each singular segment is less than $2 \pi$. A positive answer to Question $1^{\prime}$ would therefore lead to a non-rigid cone-manifold with vertices, with angle below $2 \pi$ along each singular segment. Note that Casson [C] recently gave an example of a non-rigid three-dimensional hyperbolic cone-manifold with vertices, but in which some singular segments have angles above $2 \pi$.

We do not, in this paper, give any result on Questions 1 and $1^{\prime}$. We do, however, show that some similar or related questions have negative answers. This might indicate that some ways of dealing with Questions 1 and $1^{\prime}$ are unlikely to succeed.

Questions 1 and $1^{\prime}$ can be reformulated using a duality, almost classical by now (see [R1]), between $H^{3}$ and the de Sitter space $S_{1}^{3}$, which is the unique simply connected Lorentz space with constant curvature 1. This duality (which we describe in Section 2) sends convex polyhedra in $H^{3}$ to convex space-like polyhedra in $S_{1}^{3}$, exchanging dihedral angles and edge lengths. The following is therefore equivalent to Question 1:

Question 1". Are convex space-like polyhedra in $S_{1}^{3}$, which are duals of convex hyperbolic polyhedra, determined by their edge lengths?

Note that it is a result of Rivin (see [R1], [RH], and also [M]) that those polyhedra are determined by their induced metrics, i.e., by the metrics on $S^{2}$ obtained by gluing the metrics induced on the faces of the polyhedra under consideration. When all faces are triangles, the edge lengths determine the induced metric, and therefore also the polyhedron. This leads to the (easy) result that convex polyhedra with all vertices trivalent are determined by their dihedral angles; this is the case in particular when all (exterior) dihedral angles are at least $\pi / 2$. This was a result of Andreev [An].

Some other special cases of Questions 1 have been considered, e.g., in [D1], which proves that the answer to Question $1^{\prime}$ is negative for some combinatorial structures, i.e., some combinatorial structures contain only polyhedra which are rigid. Diaz also considered some other related questions; she proved (see [D2]) that the space of dihedral angles of convex hyperbolic polyhedra is not convex. This contrasts with the results of Rivin (see [R2], and also [R3]) for ideal polyhedra: ideal polyhedra are determined by their dihedral angles, and the space of possible dihedral angles is convex. The rigidity result here is in a way not surprising since, in some sense (see [S1]) the dihedral angles of an ideal polyhedron determine its "dual metric."

Not all convex space-like polyhedra in $S_{1}^{3}$ are duals of hyperbolic convex polyhedra. Those which are so, are exactly those which do not bound a compact subset of $S_{1}^{3}$. Our first example shows that it is necessary, in Question 1", to ask for duals of hyperbolic polyhedra.

Theorem 1. There exist pairs of non-congruent convex space-like polyhedra in $S_{1}^{3}$ with the same edge lengths. 
Still in $S_{1}^{3}$, we can ask the same question as in $H^{3}$ about the dihedral angles. The answer is negative:

Theorem 2. There exist pairs of non-congruent convex space-like polyhedra in $S_{1}^{3}$ with the same dihedral angles.

Actually, this is just a consequence (using the duality between $H^{3}$ and $S_{1}^{3}$ ) of

Theorem 2'. There exist pairs of non-congruent convex polyhedra in $\mathrm{H}^{3}$ with the same edge lengths.

Similar examples exist also in $S^{3}$; the next two assertions are duals of each other by a classical duality (often called "projective") similar to that between $H^{3}$ and $S_{1}^{3}$, but between $S^{3}$ and itself.

Theorem 3. There exist pairs of non-congruent convex polyhedra in $S^{3}$ with the same dihedral angles.

Theorem 3'. There exist pairs of non-congruent convex polyhedra in $S^{3}$ with the same edge lengths.

Section 3 contains slightly more precise assertions, as well as infinitesimal versions of these theorems.

The examples in Theorems $1-3^{\prime}$ are built from a (well-known) one-parameter family of polyhedra in $\mathbf{R}^{3}$ with the same edge lengths, through a transformation discovered by Pogorelov for $H^{3}$ and $S^{3}$ (and duality to go from edge lengths to dihedral angles). This procedure does not show, however, the existence of one-parameter families of, e.g., convex hyperbolic polyhedra with the same edge lengths, but only the existence of pairs of polyhedra with the same edge lengths, and of infinitesimal deformations preserving the edge lengths.

It is possible to give analogous results in the only other non-flat three-dimensional space-form, i.e. the anti de Sitter space $H_{1}^{3}$; we have left it out since it might carry less geometrical meaning for most readers.

\section{Hyperbolic Tools}

The hyperbolic 3-space $H^{3}$ can be seen as a hypersurface of the Minkowski space $\mathbf{R}_{1}^{4}$ (with coordinates $x_{0}, x_{1}, x_{2}, x_{3}$ and norm $\|x\|^{2}=-x_{0}^{2}+x_{1}^{2}+x_{2}^{2}+x_{3}^{2}$ ) as

$$
H^{3}=\left\{x \in \mathbf{R}_{1}^{4} \mid x_{0}>0 \wedge\|x\|^{2}=-1\right\} .
$$

The de Sitter space is

$$
S_{1}^{3}=\left\{x \in \mathbf{R}_{1}^{4} \mid\|x\|^{2}=1\right\}
$$


It is a symmetric space (it can be written as $S O(4,1) / S O(3))$ and its isometry group is transitive on orthonormal frames. All its space-like totally geodesic 2-planes are therefore isometric, and they are isometric copies of $S^{2}$.

Let $p$ be an oriented 2-plane in $H^{3}$. Then $p$ is the intersection with $H^{3} \subset \mathbf{R}_{1}^{4}$ of some oriented 3-plane $q$ of $\mathbf{R}_{1}^{4}$ going through 0 . Call $q^{*}$ the oriented line orthogonal to $q$ at 0 , and $p^{*}$ the (unique) intersection of $q^{*}$ with $S_{1}^{3}$ which is on the positive side from 0. Similarly, if $p$ is a space-like 2-plane in $S_{1}^{3}$, it is the intersection with $S_{1}^{3}$ of some 3-plane $q$ containing 0 ; call $p^{*}$ the intersection with $H^{3}$ of the line orthogonal to $q$ at 0 .

Given a convex polyhedron $P$ in $H^{3}$, we can now define its dual $P^{*}$, which is a convex space-like polyhedron in $S_{1}^{3}$ : the vertices of $P^{*}$ are the duals of the 2-planes in $H^{3}$ containing the faces of $P$, and the faces of $P^{*}$ are (contained in) the 2-planes dual to the vertices of $P$. The same is obviously true for the vertices (resp. faces) of $P$ relative to the faces (resp. vertices) of $P^{*}$. Each edge of $P^{*}$ is associated to an edge of $P$, and the dihedral angle of one is the length of the other (see [RH] and [S1]).

The same kind of duality exists, but as a "self-duality," in $S^{3}$ and in the anti de Sitter space $H_{1}^{3}$. It is defined as above, but by considering $S^{3}$ in $\mathbf{R}^{4}$ in the canonical way, and $H_{1}^{3}$ in $\mathbf{R}_{2}^{4}$ as

$$
H_{1}^{3}=\left\{x \in \mathbf{R}_{2}^{4} \mid\langle x, x\rangle=-1\right\}
$$

and also, changing the sign of the metric on $H_{1}^{3}$, as

$$
H_{1}^{3} \simeq\left\{x \in \mathbf{R}_{2}^{4} \mid\langle x, x\rangle=1\right\} .
$$

Considering $H^{3}$ as a submanifold of $\mathbf{R}_{1}^{4}$ also leads to the classical "projective"-or "Klein"-model of $H^{3}$, namely:

Proposition 1. There exists a mapping $\rho: H^{3} \rightarrow B^{3}$, where $B^{3}$ is the unit ball in $\mathbf{R}^{3}$, sending each geodesic segment of $H^{3}$ to a geodesic segment of $B^{3}$.

$\rho$ can be defined by sending each point of $H^{3} \subset \mathbf{R}_{1}^{4}$ to the unit ball

$$
B^{3} \subset \mathbf{R}^{3} \simeq\left\{x \in \mathbf{R}_{1}^{4} \mid x_{0}=1\right\}
$$

along the direction of 0 . This map is clearly projective, because the geodesics of $H^{3}$ are the intersections with $H^{3}$ of 2-planes containing 0 in $\mathbf{R}_{1}^{4}$, so they are sent to segments in $B^{3}$.

In the same way, we define a projective model of a hemisphere of $S_{1}^{3}$ :

Proposition 2. There exists a mapping $\tilde{\rho}: S_{1,+}^{3} \rightarrow \mathbf{R}_{1}^{3}$, where

$$
S_{1,+}^{3}:=\left\{x \in \mathbf{R}_{1}^{4} \mid\|x\|^{2}=1 \wedge x_{1}>0\right\}
$$

sending each geodesic segment of $S_{1,+}^{3}$ to a geodesic segment of $\mathbf{R}_{1}^{3}$.

This mapping is built as for Proposition 1, by projecting in the direction of 0 on the tangent plane to $S_{1}^{3} \subset \mathbf{R}_{1}^{4}$ at a point. The same construction works in $S^{3}$ and in $H_{1}^{3}$. 
Pogorelov $[\mathrm{P}]$ has found a remarkable extension of $\rho$ :

Proposition 3. There exists a mapping $\Phi: H^{3} \times H^{3} \rightarrow \mathbf{R}^{3} \times \mathbf{R}^{3}$ such that:

(1) the restriction of $\Phi$ to the diagonal $\Delta \subset H^{3} \times H^{3}$ corresponds to $\rho$ (its image is in the diagonal $\Delta^{\prime} \subset \mathbf{R}^{3} \times \mathbf{R}^{3}$ );

(2) if $g_{1}, g_{2}:[0,1] \rightarrow H^{3}$ are geodesic segments parametrized at the same speed, and if $p_{1}, p_{2}$ are the projections of $\mathbf{R}^{3} \times \mathbf{R}^{3}$ on the two factors, then $p_{1} \circ \Phi \circ$ $\left(g_{1}, g_{2}\right)$ and $p_{2} \circ \Phi \circ\left(g_{1}, g_{2}\right)$ are geodesic segments parametrized at the same speed;

(3) there exists a point $x_{0}=\rho^{-1}(0) \in H^{3}$ such that, for each 2-plane $p \subset H^{3}$ containing $x_{0}$,

$$
\forall x \in p, \quad \forall y \in H^{3}, \quad\left(p_{1} \circ \Phi\right)(x, y) \in \rho(p) .
$$

The proof can be found in [P], except for point (3), which is obvious from the explicit definition of $\Phi$ given there. The reader may also find a (different) proof, some explanations of its existence, and some extensions in [S1].

Note that a mapping $\alpha: H^{3} \rightarrow H^{3}$ is an isometry if and only if, for each geodesic segment $g:[0,1] \rightarrow H^{3}, \alpha \circ g$ is also a geodesic segment parametrized at the same speed. Applying point (2) of Proposition 3 shows that $p_{1} \circ \Phi \circ(g, \alpha \circ g)$ and $p_{2} \circ \Phi \circ(g, \alpha \circ g)$ are then also geodesic segments parametrized at the same speed. Since this is true for any $g$, there exists an isometry $\beta: \mathbf{R}^{3} \rightarrow \mathbf{R}^{3}$ such that,

$$
\forall x \in H^{3}, \quad p_{1} \circ \Phi(x, \alpha(x))=\beta \circ p_{2} \circ \Phi(x, \alpha(x)),
$$

which says in its way that $\Phi$ "commutes with isometries" between the two factors on each side.

We can linearize $\Phi$ in the neighborhood of $\Delta$, to obtain:

Proposition 4. There exists a bundle map $\varphi: T H^{3} \rightarrow T \mathbf{R}^{3}$ such that:

(1) if $v \in T_{x} H^{3}$, then $\varphi(x, v) \in T_{\rho(x)} \mathbf{R}^{3}$;

(2) if $g:[0,1] \rightarrow H^{3}$ is a geodesic segment, and $v$ is a Jacobi field on $g$ preserving the parametrization, $\varphi(v)$ is a Jacobi field preserving the parametrization on $(\rho \circ g)$;

(3) for each 2-plane $p \subset H^{3}$ containing $x_{0}$,

$$
\forall x \in p, \quad \forall v \in T_{x} p, \quad \varphi(v) \in T_{\rho(x)} \rho(p) .
$$

Again, the only non-classical point is (3), which is obvious from point (3) of Proposition 3, and also from the following explicit definition of $\varphi$ in the projective model $\rho$ centered at $x_{0}: \varphi$ acts as $\rho_{*}$ on the 2-plane orthogonal to the radial direction at each point, and acts on the radial directions so as to preserve the norm of the radial vectors (the hyperbolic norm of a radial vector is the same as the Euclidean norm of its image). 
This Pogorelov transformation works in the same way if we replace $H^{3}$ by a hemisphere of $S^{3}$ (see [P]), or by $S_{1,+}^{3}$, or by a hemisphere of $H_{1}^{3}$ (see [LS] and [S2]). For instance, in the de Sitter case, we have:

Proposition 5. There exists a mapping $\tilde{\Phi}: S_{1,+}^{3} \times S_{1,+}^{3} \rightarrow \mathbf{R}_{1}^{3} \times \mathbf{R}_{1}^{3}$ such that:

(1) the restriction of $\tilde{\Phi}$ to the diagonal $\Delta \subset S_{1,+}^{3} \times S_{1,+}^{3}$ corresponds to $\tilde{\rho}$ (its image is in the diagonal $\left.\Delta^{\prime} \subset \mathbf{R}_{1}^{3} \times \mathbf{R}_{1}^{3}\right)$;

(2) if $g_{1}, g_{2}:[0,1] \rightarrow S_{1,+}^{3}$ are geodesic segments parametrized at the same speed, and if $\tilde{p}_{1}, \tilde{p}_{2}$ are the projections of $\mathbf{R}_{1}^{3} \times \mathbf{R}_{1}^{3}$ on the two factors, then $\tilde{p}_{1} \circ \tilde{\Phi} \circ\left(g_{1}, g_{2}\right)$ and $\tilde{p}_{2} \circ \tilde{\Phi} \circ\left(g_{1}, g_{2}\right)$ are geodesic segments parametrized at the same speed;

(3) there exists a point $y_{0}=\tilde{\rho}^{-1}(0) \in S_{1,+}^{3}$ such that, for each 2-plane $p \subset S_{1,+}^{3}$ containing $y_{0}$,

$$
\forall x \in p, \quad \forall y \in S_{1,+}^{3}, \quad\left(\tilde{p}_{1} \circ \tilde{\Phi}\right)(x, y) \in \tilde{\rho}(p) .
$$

Linearizing this leads to:

Proposition 6. There exists a bundle map $\varphi t: T S_{1,+}^{3} \rightarrow T \mathbf{R}_{1}^{3}$ such that:

(1) if $v \in T_{x} S_{1,+}^{3}$, then $\varphi t(x, v) \in T_{\tilde{\rho}(x)} \mathbf{R}_{1}^{3}$;

(2) if $g:[0,1] \rightarrow S_{1,+}^{3}$ is a geodesic segment, and $v$ is a Jacobi field on $g$ preserving the parametrization, then $\varphi t(v)$ is a Jacobi field on $(\tilde{\rho} \circ g)$ preserving the parametrization;

(3) there exists a point $x_{0}$ such that, for each 2-plane $p \subset S_{1,+}^{3}$ containing $x_{0}$,

$$
\forall x \in p, \quad \forall v \in T_{x} p, \quad \varphi t(v) \in T_{\tilde{\rho}(x)} \tilde{\rho}(p) .
$$

\section{Construction of Examples}

All our examples are built from the prism, which can be deformed in $\mathbf{R}^{3}$ keeping its edge lengths fixed. More precisely, for $a, b, c \in \mathbf{R}_{+}^{*}$ with $c<2 b$ and $u$ small enough ( $u \in(-\varepsilon, \varepsilon$ ) where $\varepsilon$ depends on $a, b, c)$ there exist $x, y \in \mathbf{R}_{+}$such that the convex polyhedron $P_{a, b, c ; u}$ with vertices of coordinates $(0,0,0),(0,0, a),(x, y, u),(x, y, u+$ $a),(x,-y, u),(x,-y, u+a)$ has all its edges of length $a, b$ or $c$. The only conditions are that $2 y=c$ and that $x^{2}+y^{2}+u^{2}=b^{2}$. The same construction works in $\mathbf{R}_{1}^{3}$, with the three edges of lengths $a$ time-like, and the other edges space-like.

Now we can use this example in $\mathbf{R}^{3}$ to prove Theorem $2^{\prime}$ : choose $(a, b, c)$ small enough so that the polyhedron $P_{a, b, c ; 0} \subset B^{3}$. Consider $P_{a, b, c ; u}$ as the image of a polyhedral immersion $\alpha_{u}$ of a fixed abstract prism $P$ into $B^{3}$, with $\alpha_{u}$ inducing the same metric on each edge of $P$ for all $u$, and $\alpha_{u}$ linear on each face of $P$ (this is possible since the $P_{a, b, c ; u}$ have the same edge lengths for fixed $\left.a, b, c\right)$.

Now apply the inverse of the Pogorelov mapping $\Phi: H^{3} \times H^{3} \rightarrow \mathbf{R}^{3} \times \mathbf{R}^{3}$ to the pair $\left(\alpha_{0}, \alpha_{u}\right)$ for a fixed $u \neq 0$. This is possible for $u$ small enough, because $\Phi_{\mid \Delta}=\rho$ and $P_{a, b, c ; 0} \subset B^{3}=\rho\left(H^{3}\right)$, so that $\left(\alpha_{0}, \alpha_{u}\right)(P) \subset \operatorname{Im}(\Phi)$. Call $q_{1}, q_{2}$ the projections 
of $H^{3} \times H^{3}$ on the two factors, and set

$$
\beta_{1}:=q_{1} \circ \Phi^{-1} \circ\left(\alpha_{0}, \alpha_{u}\right), \quad \beta_{2}:=q_{2} \circ \Phi^{-1} \circ\left(\alpha_{0}, \alpha_{u}\right) .
$$

$\beta_{1}, \beta_{2}$ are sending $P$ into $H^{3}$; we have to check that they are polyhedral, convex, and induce the same edge lengths on $P$.

Now assertion (2) of Proposition 3 shows that all edges of $P$ are sent by $\beta_{1}$ and $\beta_{2}$ to geodesic segments of the same lengths. Moreover, the two triangular faces of $P$ and the (unique) rectangular face with edge lengths $a$ and $c$ (for the metric induced by $\alpha_{0}$ ) are isometric for the metrics induced by $\alpha_{0}$ and $\alpha_{u}$; therefore, any geodesic segment on one of those faces is sent by $\alpha_{0}, \alpha_{u}$ to two geodesic segments parametrized at the same speed, and, by assertion (2) of Proposition 3, it is sent by $\beta_{1}, \beta_{2}$ to geodesic segments. This shows that $\beta_{1}$ and $\beta_{2}$ send thoses faces to totally geodesic planes in $H^{3}$.

This line of reasoning does not show that the other two rectangular faces of $P$ (with edge lengths $a$ and $b$ ) are sent by $\beta_{1}, \beta_{2}$ to totally geodesic 2-planes. This follows, however, from point (3) of Proposition 3, because both those faces contain $x_{0}$. Therefore, $\beta_{1}$ and $\beta_{2}$ are polyhedral maps. Now from the remark following Proposition 3, $\beta_{1}$ and $\beta_{2}$ are not congruent, otherwise the isometry sending one to the other would come from an isometry sending $\alpha_{0}$ to $\alpha_{u}$ in $\mathbf{R}^{3}$.

$\alpha_{0}(P)$ is convex and $\Phi_{\mid \Delta}=\rho$ is projective; taking $u=0$ would lead to $\beta_{1}=\beta_{2}$ with $\beta_{1}(P)$ convex. If $u$ is small enough, $\beta_{1}(P)$ and $\beta_{2}(P)$ are still convex, and this finishes the proof of Theorem $2^{\prime}$. Theorem 2 is a consequence using the duality in Section 2.

The same construction can be used in $S_{1}^{3}$ (with Proposition 5 instead of Proposition 3 ) and leads to Theorem 1 . It also works in $S^{3}$, and gives Theorem $3^{\prime}$ and, using the projective duality in $S^{3}$, Theorem 3 .

Note that we cannot obtain by this method pairs of non-congruent space-like polyhedra in $S_{1}^{3}$ with the same edge lengths which are duals of hyperbolic polyhedra, because $\tilde{\rho}$ is only defined on $S_{1,+}^{3}$, which does not contain any polyhedron dual of a hyperbolic polyhedron. It is possible to define a projective model and a Pogorelov transformation on a domain of $S_{1}^{3}$ containing such polyhedra, but they have no "center" (i.e., 0 is not in the image of this projective model) so that the construction above does not apply.

Repeating the proof above but for infinitesimal deformations, and using Propositions 4 and 6 instead of 3 and 5, leads to the following:

Theorem 1*. There exists a convex space-like polyhedron in $S_{1}^{3}$ which admits a nontrivial infinitesimal deformation preserving its edge lengths.

Theorem 2*. There exists a convex space-like polyhedron in $S_{1}^{3}$ which admits a nontrivial infinitesimal deformation preserving its dihedral angles.

Theorem $2^{* *}$. There exists a convex polyhedron in $H^{3}$ which admits a non-trivial infinitesimal deformation preserving its edge lengths. 
Theorem 3*. There exists a convex polyhedron in $S^{3}$ which admits a non-trivial infinitesimal deformation preserving its dihedral angles.

Theorem $3^{* *}$. There exists a convex polyhedron in $S^{3}$ which admits a non-trivial infinitesimal deformation preserving its edge lengths.

We have actually found much more than one example in each case; for each value of $a, b, c$ (which can be chosen anywhere in some open subset) we could have used the polyhedra $P_{a, b, c ; u}$ and $P_{a, b, c ; v}$ for any choice of $u, v$ small enough. This provides us with a two-parameter family of pairs of polyhedra such that, in each pair, both polyhedra have the same edge length/dihedral angles.

A geometric interpretation of this can be given in the space $\mathcal{P}$ of convex polyhedra $P$ with six vertices in $H^{3}$ (for instance). By a well-known result of Aleksandrov [Al], $\mathcal{P}$ can be identified with the space $\mathcal{M}$ of metrics on $P$ with curvature -1 outside the six vertices, where the singular curvature is strictly positive. $\mathcal{P}$ has dimension 12 and contains a codimension 2 submanifold $\mathcal{S}$ of polyhedra which are combinatorially prisms. There is also a submersion $s: U \rightarrow \mathbf{R}^{10}$, where $U$ is a neighborhood of $\mathcal{S}$ in $\mathcal{P}$, sending a polyhedron to the lengths of the edges of the prism (that is, forgetting those edges which appear when deforming a polyhedron away from $\mathcal{S}$ ). Then $s$ is neither transverse to $\mathcal{S}$ (this corresponds to the infinitesimal statement) nor even one-to-one in a neighborhood of any of the polyhedra $\rho^{-1}\left(P_{a, b, c ; u}\right)$.

\section{Acknowledgments}

This paper has benefited from important remarks from Igor Rivin, as well as from anonymous referees.

\section{References}

[Al] A. D. Aleksandrov. Convex Polyhedra. GITTL, Moskow-Leningrad, 1951. Russian; Konvexe Polyeder. Akademie-Verlag, Berlin, 1958. German.

[An] E. M. Andreev. Convex polyhedra in Lobacevskii space. Mat. Sb. (N.S.), 81(123):445-478, 1970.

[C] A. Casson. An example of weak non-rigidity for cone manifolds with vertices. Notes of a talk, Third MSJ Regional Workshop, Tokyo, July 1998.

[D1] R. Díaz. A characterization of Gram matrices of polytopes. Discrete Comput. Geom. 21(4) (1999), 581-601.

[D2] R. Díaz. Non-convexity of the space of dihedral angles of hyperbolic polyhedra. C. R. Acad. Sci. Paris Sér. I Math., 325(9):993-998, 1997.

[HK] C. D. Hodgson and S. P. Kerckhoff. Rigidity of hyperbolic cone-manifolds and hyperbolic Dehn surgery. J. Differential Geom., 48:1-60, 1998.

[LS] F. Labourie and J.-M. Schlenker. Surfaces convexes fuchsiennes dans les espaces lorentziens à courbure constante. Prépublication 96-05, Université de Paris-Sud, 1996.

[M] A. D. Milka. Unique determination of convex surfaces in pseudo-riemannian spherical space I. Ukrain. Geom. Sb., 29:113-118, 1986.

[P] A. V. Pogorelov. Extrinsic Geometry of Convex Surfaces. Translations of Mathematical Monographs, Vol. 35. American Mathematical Society, Providence, RI, 1973.

[R1] I. Rivin. Geometry of Polyhedra in Hyperbolic 3-space. Ph.D. thesis, Princeton University, 1986. 
[R2] I. Rivin. On the geometry of ideal polyhedra in hyperbolic 3-space. Topology, 32:87-92, 1993.

[R3] I. Rivin. Euclidean structures on simplicial surfaces and hyperbolic volume. Ann. of Math., 139:553-580, 1994.

[RH] I. Rivin and C. D. Hodgson. A characterization of compact convex polyhedra in hyperbolic 3-space. Invent. Math., 111:77-111, 1993.

[S1] J.-M. Schlenker. Métriques sur les polyèdres hyperboliques convexes. J. Differential Geom., 48(2):323$405,1998$.

[S2] J.-M. Schlenker. Représentations de surfaces hyperboliques complètes dans $H^{3}$. Ann. Inst. Fourier, 48(3):837-860, 1998.

Received November 16, 1998, and in revised form March 8, 1999. 